\title{
Second Order Necessary Condition in Major Constraints Multi-Objective Programming
}

\author{
Xuanwei Zhou* \\ School of Basic Courses, Zhejiang Shuren University, Hangzhou 310015, China \\ *Corresponding author
}

\begin{abstract}
Second order necessary optimality condition in multi-objective programming with major constraints is studied. With the aid of the major constraints set and its structure representation, a second order necessary condition for major constrains local Pareto weakly efficient solution is given under a weakly convex inclusion condition and some constraint qualifications. The result develops the theory of multi-objective programming and is useful in the design of numerical methods in the major constraints multi-objective programming.
\end{abstract}

Keywords-major constraints multi-objective programming; major constraints Pareto weakly efficient solution; second order necessary condition

\section{INTRODUCTION}

Multi-objective programming(MOP) is an important part of mathematical programming, and its theory and methods have become practical tool in modern quantitative decision-making. In view of the fact that the decision-makers usually give various constraint conditions under their different needs in the establishment of a practical multi-objective programming model, so the inequality constraints in the given model are often incompatible. For this reason, we consider a kind of MOP problem called as major constraints(MC) MOP. Its major inequality constraints are compatible.

In Ref.[1], the concepts of major cone and major order were introduced, and a kind of MC problem was presented. In Ref.[2], the MC programming problem was studied and the optimality conditions of MC optimal solutions were proved by using the major constraints set and its structure representation and some constraint qualification conditions. Then, the result of Ref.[2] was extended to MCMOP in Ref.[3].

The study of optimality condition(OC) is always a central subject of mathematical programming theory, which is the basis of numerical method design and is indispensable to other theories such as sensitivity analysis and stability analysis. Since the 1940s, due to the success of mathematical programming theory and methods in solving various practical problems, the study of its first-order and second-order as well as higher-order optimality conditions have received extensive attention (see Ref. [4]-[17] ).

In paper introduce the concept of weakly convex inclusion. With the aid of the structure representation of $\mathrm{MC}$ set, a second order necessary OC for the MC local Pareto weakly efficient solution is obtained when the objective vector function satisfies the weakly convex inclusion condition.

\section{SECOND ORDER NECESSARY CONDITION}

Let $f$ from $R^{n}$ to $R^{m}$ and $g$ from $R^{n}$ to $R^{p}$ be nonlinear vector functions. The general nonlinear MOP is:

$$
\begin{cases}\min & f(x), \\ \text { s.t. } & g(x) \leq 0 .\end{cases}
$$

Denote $g(x)=\left(g_{1}(x), \cdots, g_{p}(x)\right)^{T}$, then the constraint conditions in (NMOP) are

$$
g_{i}(x) \leq 0 \quad(i \text { from1 to } p)
$$

In practical application, the above each inequality $g_{i}(x) \leq 0$ ( $i$ from 1 to $p$ ) is often given from decisionmakers independently. It may sometimes occur that the inequality system is not mutually compatible. In this regard, the author (see Ref.[1]) proposed MC nonlinear programming problem under the help of major cone. Based on the results of Ref.[1]-[3], this paper considers the following problem called major constraints multi-objective programming (MCMOP):

$$
\begin{cases}\min & f(x)=\left(f_{1}(x), \cdots, f_{m}(x)\right) \\ \text { s.t. } & g(x)=\left(g_{1}(x), \cdots, g_{p}(x)\right) \leq 0\end{cases}
$$

(MCMOP)

Where the major constraints condition $\left(g_{1}(x), \cdots, g_{p}(x)\right) \underset{c l H}{\leq} 0$ of problem (MCMOP) means that there exist at least $\left[\frac{p+1}{2}\right]$ numbers $i_{1}, \cdots, i_{\left[\frac{p+1}{2}\right]}$ belong to $\{1, \cdots, p\}$ such the condition that $x$ satisfies the inequality 


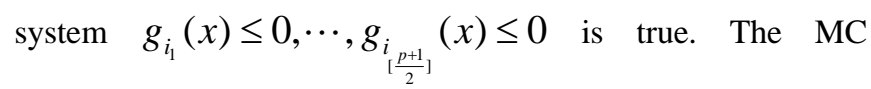
set $X_{M}$ is denoted as $X_{M}=\{x \mid g(x) \underset{c l H}{\leq 0} 0\}$.

Definition 1 Let $f$ from $R^{n}$ to $R^{m}$ and $g$ from $R^{n}$ to $R^{p}$ be nonlinear vector functions. If $x^{*} \in X_{M}$ and there exists an $\delta>0$ such that there is not $x \in X_{M} \cap N_{\delta}\left(x^{*}\right)$ satisfying

$$
f_{k}(x)<f_{k}\left(x^{*}\right)(k \text { from } 1 \text { to } m)
$$

where $\quad N_{\delta}\left(x^{*}\right)=\left\{x \in R^{n}\left\|x-x^{*}\right\|<\delta\right\} \quad$ is $\quad$ a neighborhood of $x^{*}$, then $X^{*}$ is said to be a MC local Pareto weakly efficient solution(WES). The set of all MC local Pareto WES is denoted by $S_{M}^{L}$.

In order to give a second-order necessary OC of (MCMOP), we need the following results from Ref. [2].

Let $H \subset R^{p}$ be the major cone. Then we have

$$
\begin{gathered}
\bar{H}=\left\{v \in R^{p} \|\left. v\right|_{+} \geq|v|_{-}\right\} \\
=\left\{x \in R^{n} \mid-g(x) \in K_{i_{1}, \cdots, i_{\left[\frac{p+1}{2}\right]}}\right\},
\end{gathered}
$$

where $K_{i_{1}, \cdots, i_{\left[\frac{p+1}{2}\right]}}=\left\{v \in R^{p} \mid v_{i_{1}} \geq 0, \cdots, v_{i_{\left[\frac{p+1}{2}\right]}} \geq 0\right\}$ for any $i_{1}, \cdots, i_{\left[\frac{p+1}{2}\right]}$ belong to $\{1, \cdots, p\}$.

Denote

$$
X_{i_{1}, \cdots, i_{\left[\frac{p+1}{2}\right]}}=\left\{x \in R^{n} \mid-g(x) \in K_{i_{1}, \cdots, i_{\left[\frac{p+1}{2}\right]}}\right\} .
$$

Then the structure of the MC set $X_{M}$ is represented by

$$
X_{M}=\bigcup X_{i_{1}, \cdots, i_{\left[\frac{p+1}{2}\right]}}
$$

for all $i_{1}, \cdots, i_{\left[\frac{p+1}{2}\right]}$ belong to $\{1, \cdots, p\}$.

Lemma1 Let $h$ from $R^{n}$ to $R$ and $g$ from $R^{n}$ to $R^{p}$ be continuously differentiable at $X^{*} \in X_{M}$ and let $X^{*} \in S_{M}^{L}$. For any $i_{1}, \cdots, i_{\left[\frac{p+1}{2}\right]}$ belong to $\{1, \cdots, p\}$, if $x^{*} \in X_{i_{1}, \cdots, i_{\left[\frac{p+1}{2}\right]}}$ and $g_{i_{1}}(x), \cdots, g_{\left[\frac{p+1}{2}\right]}(x)$ have K-T constraint qualification at $X^{*}$, then there exists $\left(\tilde{\lambda}_{i_{1}}, \cdots, \tilde{\lambda}_{i_{\left[\frac{p+1}{2}\right]}}\right) \in R^{\left[\frac{p+1}{2}\right]}$ satisfying

$$
\begin{gathered}
\nabla h\left(x^{*}\right)+\sum_{m=1}^{\left[\frac{p+1}{2}\right]} \tilde{\lambda}_{i_{m}} \nabla g_{i_{k}}\left(x^{*}\right)=0 \\
\tilde{\lambda}_{i_{m}} g_{i_{m}}\left(x^{*}\right)=0, m=1,2, \cdots,\left[\frac{p+1}{2}\right] .
\end{gathered}
$$

Definition 2 Let $X^{*} \in S_{M}^{L}$. For any $i_{1}, \cdots, i_{\left[\frac{p+1}{2}\right]}$ belong to $\quad\{1, \cdots, p\} \quad, \quad$ if $\quad x^{*} \in X_{i_{1}, \cdots, i_{\left[\frac{p+1}{2}\right]}} \quad$, Denoting $F_{i_{1}, \cdots, i_{\left[\frac{p+1}{2}\right]}}=f\left(X_{i_{1}, \cdots, i_{\left[\frac{p+1}{2}\right]}}\right)$, and there exist a convex set $H_{i_{1}, \cdots, i_{\left[\frac{p+1}{2}\right]}}$ and a neighborhood $N$ of $f\left(x^{*}\right)$ meeting

$$
\begin{aligned}
& \text { 1) } F_{i_{1}, \cdots, i_{\left[\frac{p+1}{2}\right]}} \cap N \subset H_{i_{1}, \cdots, i_{\left[\frac{p+1}{2}\right]}} . \\
& \text { 2) } H_{i_{1}, \cdots, i_{\left[\frac{p+1}{2}\right]}} \cap\left(f\left(x^{*}\right)-R_{+}^{m}\right)=\varnothing,
\end{aligned}
$$

then we call that the objective vector function $f(x)$ satisfies the weakly convex inclusion condition at $X^{*}$.

Lemma2 Let $x^{*} \in S_{M}^{L}$ be a MC local Pareto WES of MCMP and let $f(x)$ satisfy the weakly convex inclusion condition at $x^{*}$. For any $i_{1}, \cdots, i_{\left[\frac{p+1}{2}\right]}$ belong to $\{1, \cdots, p\}$, if $X^{*} \in X_{i_{1}, \cdots, i_{\left[\frac{p+1}{2}\right]}} \quad$ then there exists $\tilde{\mu}_{i_{1}, \cdots, i_{\left[\frac{p+1}{2}\right]}}$ $=\left(\tilde{\mu}_{1}, \cdots, \tilde{\mu}_{m}\right)^{T} \in R_{+}^{m}$ such that $x^{*}$ is a local optimal solution(LOS) of the following linear weighting problem(LMP)

$$
\begin{cases}\min & \tilde{\mu}_{i_{1}, \cdots, i_{\left[\frac{p+1}{2}\right]}^{T}} f(x), \\ \text { s.t. } & x \in X_{i_{1}, \cdots, i_{\left[\frac{p+1}{2}\right]}} .\end{cases}
$$

Proof By convex sets separation theorem, this Lemma is easy to know.

Now we give the second order OC for the MC local Pareto WES of MCMOP. 
Theorem 1 Let $f$ from $R^{n}$ to $R^{m}$ and $g$ from $R^{n}$ to $R^{p}$

Definition 33 Let $S$ be a nonempty subset of $R^{n}$ and let $X^{*} \in c l S$. The tangent set to $S$ at $X^{*}$ is defined by

$T\left(x^{*}, S\right)=\left\{d \mid\right.$ there exist $x^{(k)} \in S, x^{(k)} \rightarrow x^{*}$ and $t_{k}>0$ such that $\left.d=\lim _{k \rightarrow \infty}\left(x^{(k)}-\bar{x}\right) / t_{k}\right\}$.

According to Definition3, we easy see that if a sequence $\left\{x^{(k)}\right\} \subset S$ converges to $x^{*}$ satisfying

$$
\lim _{k \rightarrow \infty} \frac{x^{(k)}-x^{*}}{\left\|x^{(k)}-x^{*}\right\|}=d
$$

then $d \in T\left(x^{*}, S\right)$. For any $i_{1}, \cdots, i_{\left[\frac{p+1}{2}\right]}$ belong to $\{1, \cdots, p\}$ and $\left(\tilde{\lambda}_{i_{1}}, \cdots, \tilde{\lambda}_{\left[\frac{p+1}{2}\right]}\right) \in R^{\left[\frac{p+1}{2}\right]}$ satisfying (1), denote

$$
\begin{gathered}
I_{i_{1}, \cdots, i_{[}\left[\frac{p+1}{2}\right]}=\left\{i_{m} \in\left\{i_{1}, \cdots, i_{\left[\frac{p+1}{2}\right]}\right\} \mid g_{i_{m}}\left(x^{*}\right)=0\right\} \\
S_{i_{1}, \cdots, i_{\left[\frac{p+1}{2}\right]}}=\left\{x \in R^{n} \mid g_{i_{m}}(x)=0, i_{m} \in I_{i_{1}, \cdots, i_{\left[\frac{p+1}{2}\right]}},\right. \\
\left.\tilde{\lambda}_{i_{m}}>0 ; g_{i_{m}}(x) \leq 0, i_{m} \in I_{\left.i_{1}, \cdots, i_{\left[\frac{p}{2}\right]}\right]}, \tilde{\lambda}_{i_{m}}=0\right\} .
\end{gathered}
$$

Let the tangent cone of $S_{i_{1}, \cdots, i_{\left[\frac{p+1}{2}\right]}}$ at $X^{*}$ by $T_{i_{1}, \cdots, i_{\left[\frac{p+1}{2}\right]}}$.

Denote

$$
\begin{gathered}
G_{i_{1}, \cdots, i_{\left[\frac{p+1}{2}\right]}}=\left\{d \in R^{n} \mid \nabla g_{i_{m}}\left(x^{*}\right)^{T} d=0, i_{m} \in I_{i_{1}, \cdots, i_{\left[\frac{p+1}{2}\right]}},\right. \\
\left.\tilde{\lambda}_{i_{m}}>0 ; \nabla g_{i_{m}}\left(x^{*}\right)^{T} \leq 0, i_{m} \in I_{\left.i_{1}, \cdots, i_{\left.i \frac{p}{2}\right]}\right]}, \tilde{\lambda}_{i_{m}}=0\right\} .
\end{gathered}
$$

We easy know $G_{i_{1}, \cdots, i_{\left[\frac{p+1}{2}\right]}} \supset T_{i_{1}, \cdots, i_{\left[\frac{p+1}{2}\right]}}$, but the reverse is not true, that is, the set $G_{i_{1}, \cdots, i_{\left[\frac{p+1}{2}\right]}}$ is not necessarily contained in the tangent cone $T_{i_{1}, \cdots, i_{\left[\frac{p+1}{2}\right]}}$.

If the condition $G_{\left.i_{1}, \cdots, i^{i} \frac{p+1}{2}\right]} \subset T_{\left.i_{1}, \cdots, i_{[} \frac{p+1}{2}\right]}$ is also true, the second order OC for the local Pareto weakly efficient solution of MC programming is given below. be second order continuously differentiable at $x^{*} \in X_{M}$ and let $X^{*} \in S_{M}^{L}$. For any $\left[\frac{p+1}{2}\right]$ numbers $i_{1}, \cdots, i_{\left[\frac{p+1}{2}\right]}$ belong to $\{1, \cdots, p\}$, if $x^{*} \in X_{i_{1}, \cdots, i_{\left[\frac{p+1}{2}\right]}}, f(x)$ satisfies the weakly convex inclusion condition at $x^{*}, g_{i_{1}}(x), \cdots, g_{i_{\left[\frac{p+1}{2}\right]}}(x)$ have K-T constraint qualification at $x^{*}$, and $G_{i_{1}, \cdots, i_{\left[\frac{p+1}{2}\right]}}=T_{i_{1}, \cdots, i_{\left[\frac{p+1}{2}\right]}}$ holds, then there exists $\tilde{\mu}_{\left.i_{1}, \cdots, i_{i} \frac{p+1}{2}\right]}=\left(\tilde{\mu}_{1}, \cdots, \tilde{\mu}_{m}\right)^{T} \in R_{+}^{m}$,

$$
\tilde{\lambda}_{i_{1}, \cdots, i_{\left[\frac{p+1}{2}\right]}}=\left(\tilde{\lambda}_{i_{1}}, \cdots, \tilde{\lambda}_{i_{\left[\frac{p+1}{2}\right]}}\right) \in R^{\left[\frac{p+1}{2}\right]} \text { such that }
$$

$$
\begin{aligned}
& \tilde{\mu}_{\left.i_{1}, \cdots, i_{\left[\frac{p+1}{2}\right]}^{T}\right]} \nabla f\left(x^{*}\right)+\sum_{m=1}^{\left[\frac{p+1}{2}\right]} \tilde{\lambda}_{i_{m}} \nabla g_{i_{k}}\left(x^{*}\right)=0, \\
& \tilde{\lambda}_{i_{m}} g_{i_{m}}\left(x^{*}\right)=0, m=1,2, \cdots,\left[\frac{p+1}{2}\right]
\end{aligned}
$$

is true, and for each $d \in G_{i_{1}, \cdots, i_{\left[\frac{p+1}{2}\right]}}$, we have

$$
d^{T} \nabla_{x}^{2} L\left(x^{*}, \tilde{\lambda}_{i_{1}, \cdots, i_{\left[\frac{p+1}{2}\right]}}\right) d \geq 0 .
$$

where

$$
L\left(x, \tilde{\lambda}_{i_{1}, \cdots, i_{\left[\frac{p+1}{2}\right]}}\right)=\tilde{\mu}_{i_{1}, \cdots, i_{\left[\frac{p+1}{2}\right]}^{T}} f(x)+\sum_{m=1}^{\left[\frac{p+1}{2}\right]} \tilde{\lambda}_{i_{m}} g_{i_{m}}(x),
$$

$\nabla_{x}^{2} L\left(x^{*}, \tilde{\lambda}_{\left.i_{1}, \cdots, i_{\left[\frac{1}{2}+1\right.}^{2}\right]}\right)$ is Hesse matrix of function $L\left(x, \tilde{\lambda}_{i_{1}, \cdots, i_{\left[\frac{p+1}{2}\right]}}\right)$ at $x^{*}$.

Proof let $x^{*} \in S_{M}^{L}$ and let $x^{*} \in X_{i_{1}, \cdots, i_{\left[\frac{p+1}{2}\right]}}$ for some $\left[\frac{p+1}{2}\right]$ numbers $i_{1}, \cdots, i_{\left[\frac{p+1}{2}\right]}$ belong to $\{1, \cdots, p\}$. Since $f(x)$ satisfies the weakly convex inclusion condition at $x^{*}$, by Lemma 2 , there exists 


$$
\begin{aligned}
& +\frac{1}{2}\left(x^{(k)}-x^{*}\right)^{T} \nabla_{x}^{2} L\left(x^{*}, \tilde{\lambda}_{i_{1}, \cdots, i_{\left[\frac{p+1}{2}\right]}}\right)\left(x^{(k)}-x^{*}\right) \\
& +o\left(\left\|x^{(k)}-x^{*}\right\|^{2}\right)
\end{aligned}
$$

such that $X^{*}$ is a LOS of LWP.

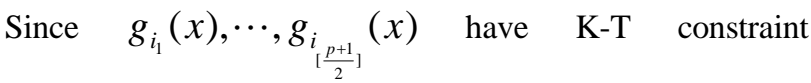
qualification at $x^{*}$, by Lemma1, there exists $\tilde{\lambda}_{i_{1}, \cdots, i_{\left[\frac{p+1}{2}\right]}}=\left(\tilde{\lambda}_{i_{1}}, \cdots, \tilde{\lambda}_{\left[\frac{p+1}{2}\right]}\right) \in R^{\left[\frac{p+1}{2}\right]}$ such that (4) holds.

Now for each $d \neq 0, d \in G_{i_{1}, \cdots,{ }_{\left[\frac{p+1}{2}\right]}}$, since $G_{i_{1}, \cdots, i_{\left[\frac{p+1}{2}\right]}}=T_{i_{1}, \cdots, i_{\left[\frac{p+1}{2}\right]}}$, it follows $d \in T_{i_{1}, \cdots, i_{\left[\frac{p+1}{2}\right]}}$. Then there exist $x^{(k)} \in S_{i_{1}, \cdots, i_{\left[\frac{p+1}{2}\right]},}, x^{(k)} \rightarrow x^{*}$ and $t_{k}>0$ such that

$$
\lim _{k \rightarrow \infty}\left(x^{(k)}-x^{*}\right) / t_{k}=d \text {. }
$$

Expanding $L\left(x, \tilde{\lambda}_{i_{1}, \cdots, i_{\left[\frac{p+1}{2}\right]}}\right)$ at $x^{*}$ by Taylor's formula and taking $X=x^{(k)}$, we have

$$
\begin{aligned}
& L\left(x^{(k)}, \tilde{\lambda}_{\left.i_{1}, \cdots, i_{[p+1}\right]}\right)=L\left(x^{*}, \tilde{\lambda}_{i_{1}, \cdots, i_{\left.\frac{p+1}{2}\right]}}\right)+\nabla_{x} L\left(x^{*}, \tilde{\lambda}_{i_{1}, \cdots, i_{\left[\frac{p+1}{2}\right.}}\right)^{T}\left(x^{(k)}-x^{*}\right) \\
& \left.\quad+\frac{1}{2}\left(x^{(k)}-x^{*}\right)^{T} \nabla_{x}^{2} L\left(x^{*}, \tilde{\lambda}_{i_{1}, \cdots, i_{\left[\frac{p+1}{2}\right]}}\right)\left(x^{(k)}-x^{*}\right)\right) \\
& \quad+o\left(\left\|x^{(k)}-x^{*}\right\|^{2}\right) .
\end{aligned}
$$

Since $x^{(k)} \in S_{i_{1}, \cdots, i_{\left[\frac{p+1}{2}\right]}}, x^{(k)} \rightarrow x^{*}$, it follows that $\tilde{\lambda}_{i_{m}}^{\tilde{i}_{1}, \cdots,{ }{ }^{\left[\frac{p+1}{2}\right]}} g_{i_{m}}\left(x^{(k)}\right)=0\left(m=1, \cdots,\left[\frac{p+1}{2}\right]\right)$. From (5) we obtain

$$
\begin{gathered}
L\left(x^{(k)}, \tilde{\lambda}_{i_{1}, \cdots, i_{\left[\frac{p+1}{2}\right]}}\right)=\tilde{\mu}_{i_{1}, \cdots, i_{\left[\frac{p+1}{2}\right]}^{T}}^{T} f\left(x^{(k)}\right) \\
L\left(x^{*}, \tilde{\lambda}_{i_{1}, \cdots, i_{\left[\frac{p+1}{2}\right]}}\right)=\tilde{\mu}_{i_{1}, \cdots, i_{\left[\frac{p+1}{2}\right]}^{T}}^{T} f\left(x^{*}\right)
\end{gathered}
$$

Substituting (7) (8) into (6), we have by (4)

$$
\tilde{\mu}_{i_{1}, \cdots, i_{\left[\frac{p+1}{2}\right]}^{T}}^{T} f\left(x^{(k)}\right)=\tilde{\mu}_{\left.i_{1}, \cdots, i_{\left[\frac{p+1}{2}\right]}^{T}\right]} f\left(x^{*}\right)
$$

Since $x^{*}$ is a LOS of LWP, there must be $\tilde{\mu}_{i_{1}, \cdots, i_{\left[\frac{p+1}{2}\right]}^{T}}^{T} f\left(x^{(k)}\right) \geq \tilde{\mu}_{i_{1}, \cdots, i_{\left[\frac{p+1}{2}\right]}^{T}}^{T} f\left(x^{*}\right)$ as $k$ sufficiently large. So from (9) for sufficiently large $k$,

$$
\begin{gathered}
\frac{1}{2}\left(x^{(k)}-x^{*}\right)^{T} \nabla_{x}^{2} L\left(x^{*}, \tilde{\lambda}_{i_{1}, \cdots, i_{\left[\frac{p+1}{2}\right]}}\right)\left(x^{(k)}-x^{*}\right) \\
+o\left(\left\|x^{(k)}-x^{*}\right\|^{2}\right) \geq 0 .
\end{gathered}
$$

Dividing both sides of the above equation by $t_{k}^{2}$ and as $k \rightarrow \infty$, then

$$
d^{T} \nabla_{x}^{2} L\left(x^{*}, \tilde{\lambda}_{i_{1}, \cdots, i_{\left[\frac{p+1}{2}\right]}}\right) d \geq 0
$$

\section{CONCLUSION}

In this paper, the inequality constraints system of the MOP is incompatible, but its major inequality constraints system is compatible. So this paper studies a new class of MOP problem. The new type of MOP is called MC multi-objective programming.

The establishment of second order OCs is very important to a MOP problem. This paper gives the concept of MC local Pareto weakly efficient solution. Then using the of MC set and its structure representation and some constraint qualification conditions, the second order necessary OC for MC local Pareto weakly efficient solution is obtained.

Then, we will further study the theory such as stability analysis and sensitivity analysis, and we will also study how to design methods for its numerical solutions and so on.

\section{REFERENCES}

[1] Y. D. Hu, “Classes of major order of vector space,” Chinese Annals of Mathematics, vol.11,1990, pp.269-280.

[2] Y. D. Hu, X. W. Zhou, "Major constraint programming and its optimality conditions, ” OR Transactions, vol. 6,2002, pp. 69-75.

[3] X. W. Zhou, "Optimality conditions of Major constraint multiobjective programming,” Mathematica Applicata,vol.29,2016, pp.902-909.

[4] H. Kawasaki, "Second-order necessary conditions of the Kuhn-Tucker type under new constraint qualification," J. Optim. Theory Appl., vol.57,1988,pp.253-264.

[5] B. Aghezzaf, M. Hachimi, "Second-order optimality conditions in multiobjective optimization problems," J. Optim. Theory Appl., vol.102,1999, pp.37-50. 
[6] M. Hackimi, B. Aghezzaf, New results on second-order optimality conditions in vector optimization problems," J. Optim. Theory Appl., vol. 135,2007, pp.117-133.

[7] M.C.Maciel, S.A.Santos, G.N.Sottosanto, "On second-order optimality conditions for vector optimization,” J. Optim. Theory Appl., vol.149,2011, pp.332-351.

[8] R.Andreani, C.E.Echagüe, M.L.Schuverdt, "Constant rank condition and second-order constraint qualification,” J. Optim. Theory Appl., vol.146, 2010, pp.255-266.

[9] E. Ning, W.Song and Y. Zhang, "Second order sufficient optimality conditions in vector optimization," Journal of Global Optimization, vol.54,2012, pp.537-549.

[10] P.Q. Khanh, N.D. Tuan, "First and second-order optimality conditions using approximations for nonsmooth vector optimization in Banach spaces,” J. Optim. Theory Appl., vol. 130,2006, pp.289-308.

[11] P. Q. Khanh, N. D. Tuan, "First and second-order approximations as derivatives of mappings in optimality conditions for nonsmooth vector optimization, ” Appl. Math. Optim., vol. 58,2008, pp.147-166.

[12] P. Q. Khanh, N. D. Tuan, "Second-order optimality conditions using approximations for nonsmooth vector optimization problems under inclusion constraints,” Nonlinear Anal., vol. 74,2011, pp. 4338-4351.

[13] J. F. Bonnans, R. Cominetti, A. Shapiro, "Second order optimality conditions based on parabolic second order tangent sets," SIAM J. Optim., vol. 9,1999, pp.466-492 .

[14] G. Bigi, "On sufficient second order optimality conditions in multiobjective optimization,” Math. Methods Oper. Res., vol. 63,2006, pp.77-85.

[15] A.Chinchuluun, P.N.Pardalos, "A survey of recent developments in multiobjective optimization,” Ann.Oper. Res., vol.154,2007, pp.29-50 .

[16] H.Gfrerer, "Second-order optimality conditions for scalar and vector optimization problems in Banach spaces," SIAM J. Control Optim., vol.45,2006,pp. 972-997

[17] I. Ginchev, V.I.Ivanov "Second-order optimality conditions for problem with C 1 data,” J. Math. Anal. Appl., vol.340,2008, pp.646-657 . 